\title{
Characteristic Distribution of the skin lesions in Tuberous Sclerosis Complex.
}

\section{Aya Takahashi1)2), Ichiro Katayama2), Manabu Fujimoto2), Wataya-Kaneda Mari2 \\ 1) Department of Regenerative Dermatology, Osaka University Graduate School of Medicine \\ 2) Department of Dermatology, Osaka University Graduate School of Medicine}

\section{Background}

Tuberous sclerosis complex (TSC) is an autosomal dominant genetic disorder, and results from mutations of either TSC1 or TSC2 gene. They encode hamartin and tuberin respectively and were discovered in the 1990s, improving the diagnosis of TSC. Either of these two gene mutations leads to constitutive activation of the mammalian/mechanistic target of rapamycin complex1 (mTORC1), resulting in a broad range of symptoms occurring at various times throughout the individual's lifetime. Hamartoma of the various organs is the hallmark of TSC.

\section{Objective}

Our purpose is to find out the cause of difference in distributions between skin manifestations, and we conducted a statistical analysis of the skin manifestations of TSC especially shagreen patch and white macule in a population and distribution.

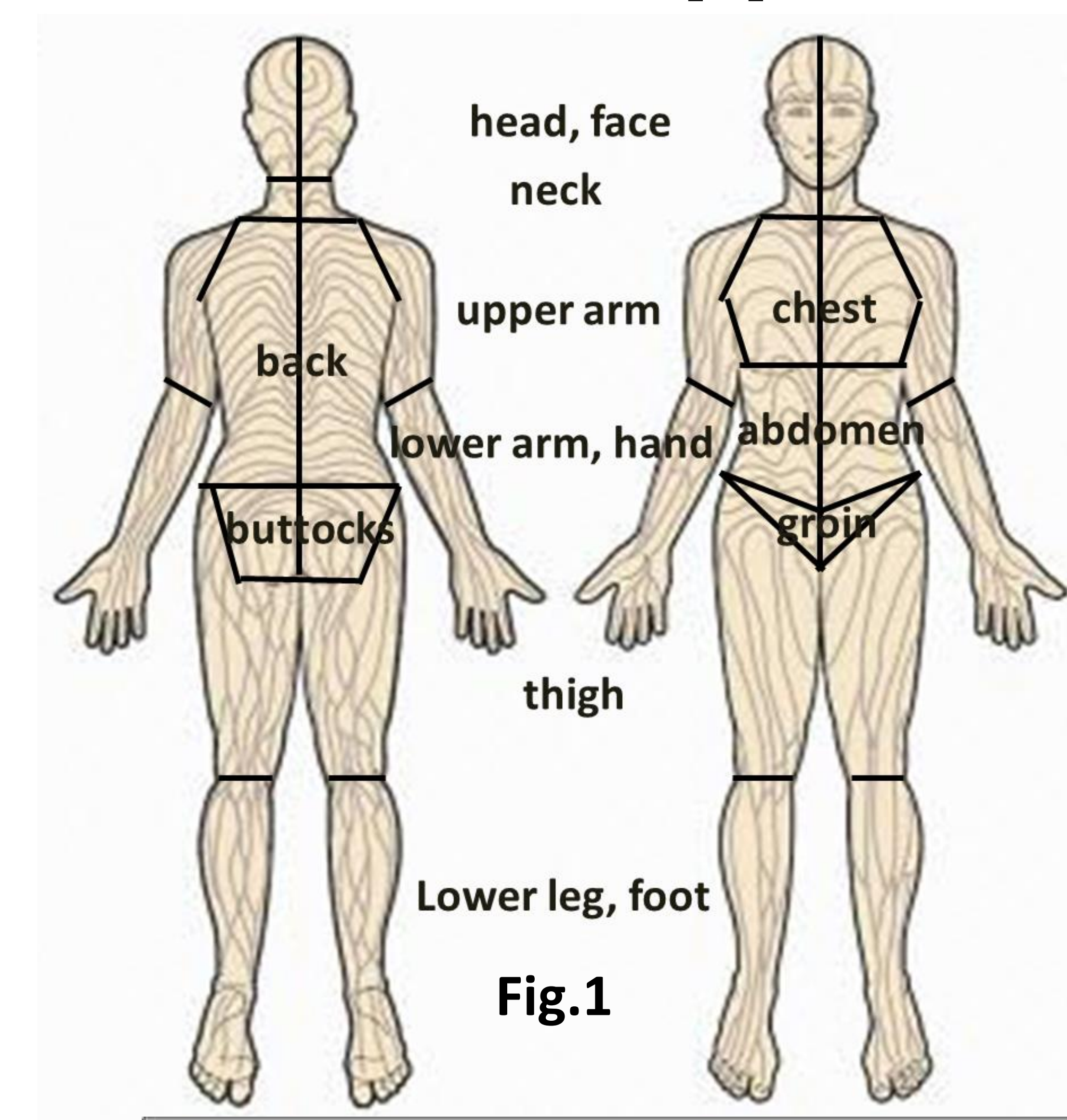

\section{Patients \& Methods}

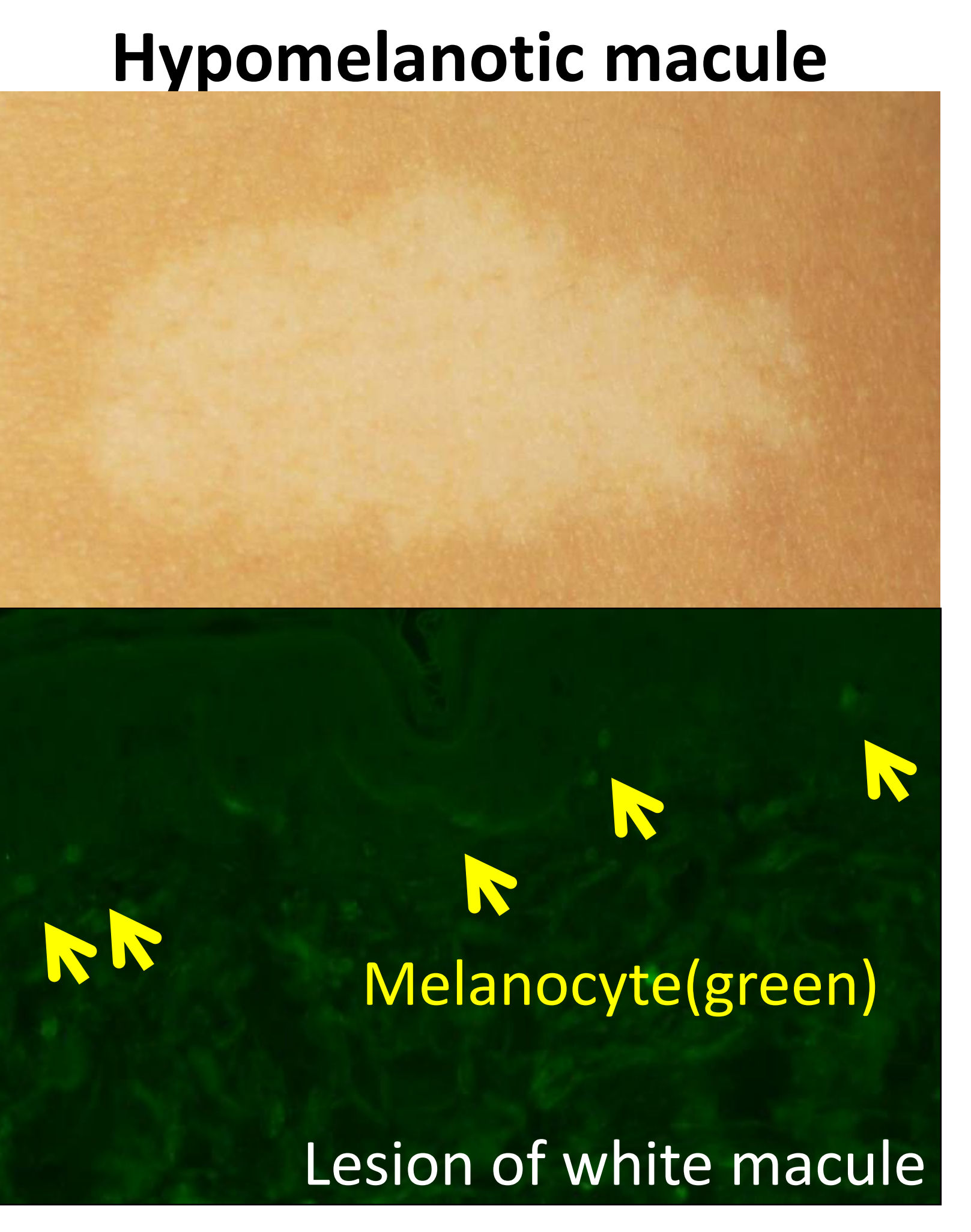

Shagreen patch

Patients All adult and child Japanese TSC patients were examined at Osaka University Hospital. They were comprised of 88 men and 124 women whose median age was 27 . They were enrolled in an observational cohort study. M88 , F124, age 27.0 00.84 years

Methods Shagreen patches and white macules were categorized per anatomic location and size. Lesions were classified as the right figure(Fig1). Size of several manifestations are based on the length of the major axis and scored. (1): $>5 \mathrm{~cm} \cdot-3$ points (2): 1 to $<5 \mathrm{~cm} \cdot-2$ points (3): $<1 \mathrm{~cm} \cdot-1$ point $\left.^{2}\right)$

The distributions are obtained for the product of the size and the numbers of the manifestation Ex) $3 \mathrm{~cm}$ and two $6 \mathrm{~cm}$ white macules are in right back: 2 points +3 poits $\times 2=8$ points(in right back)

\section{Result}

\section{(1)The site of predilection for both manifestations was upper and lower back} and were no laterality of their distribution.

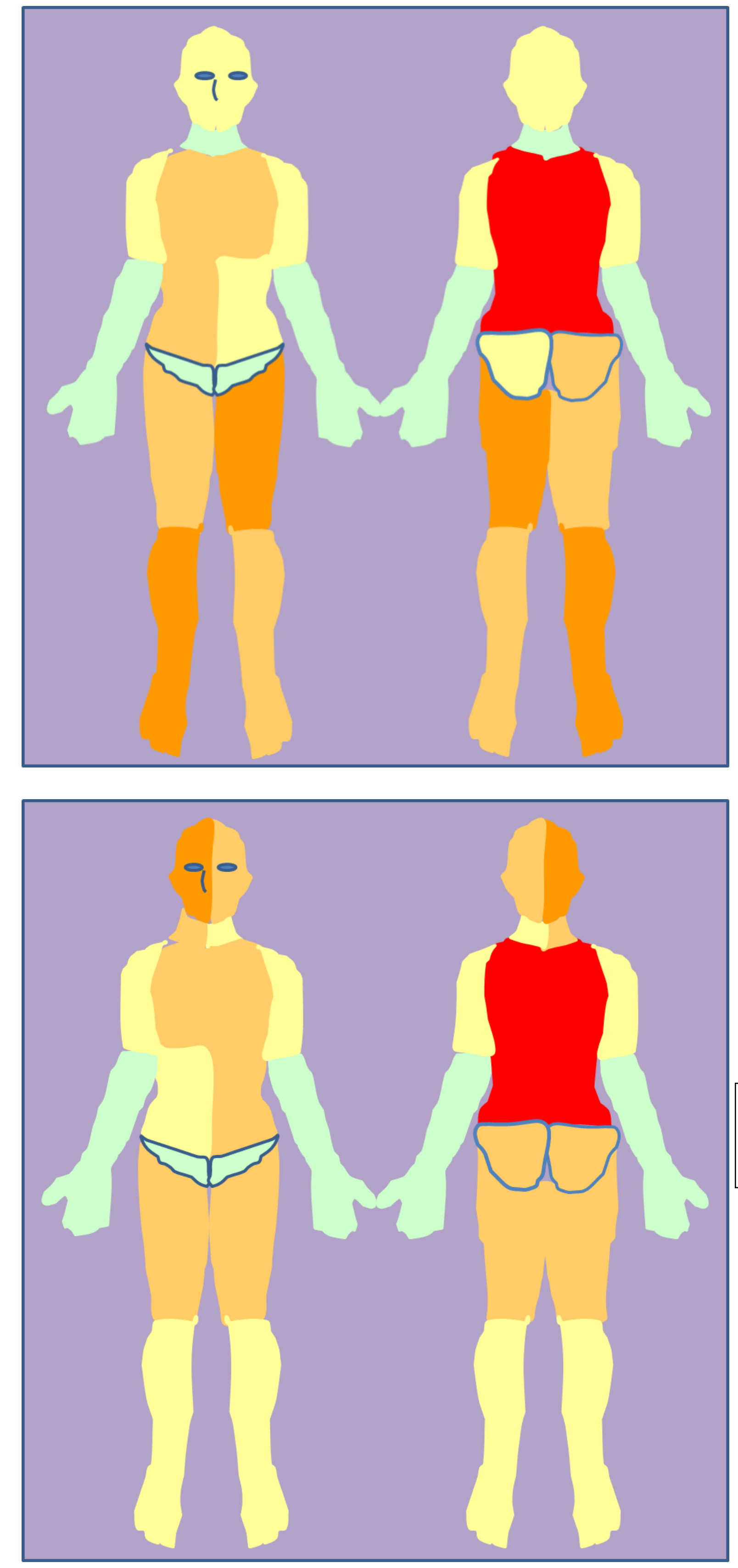

White macule

30 points $>$

$30 \sim 50$

$50 \sim 100$

$100 \sim 150$ $150_{\text {ponts }}<$

Shagreen patch

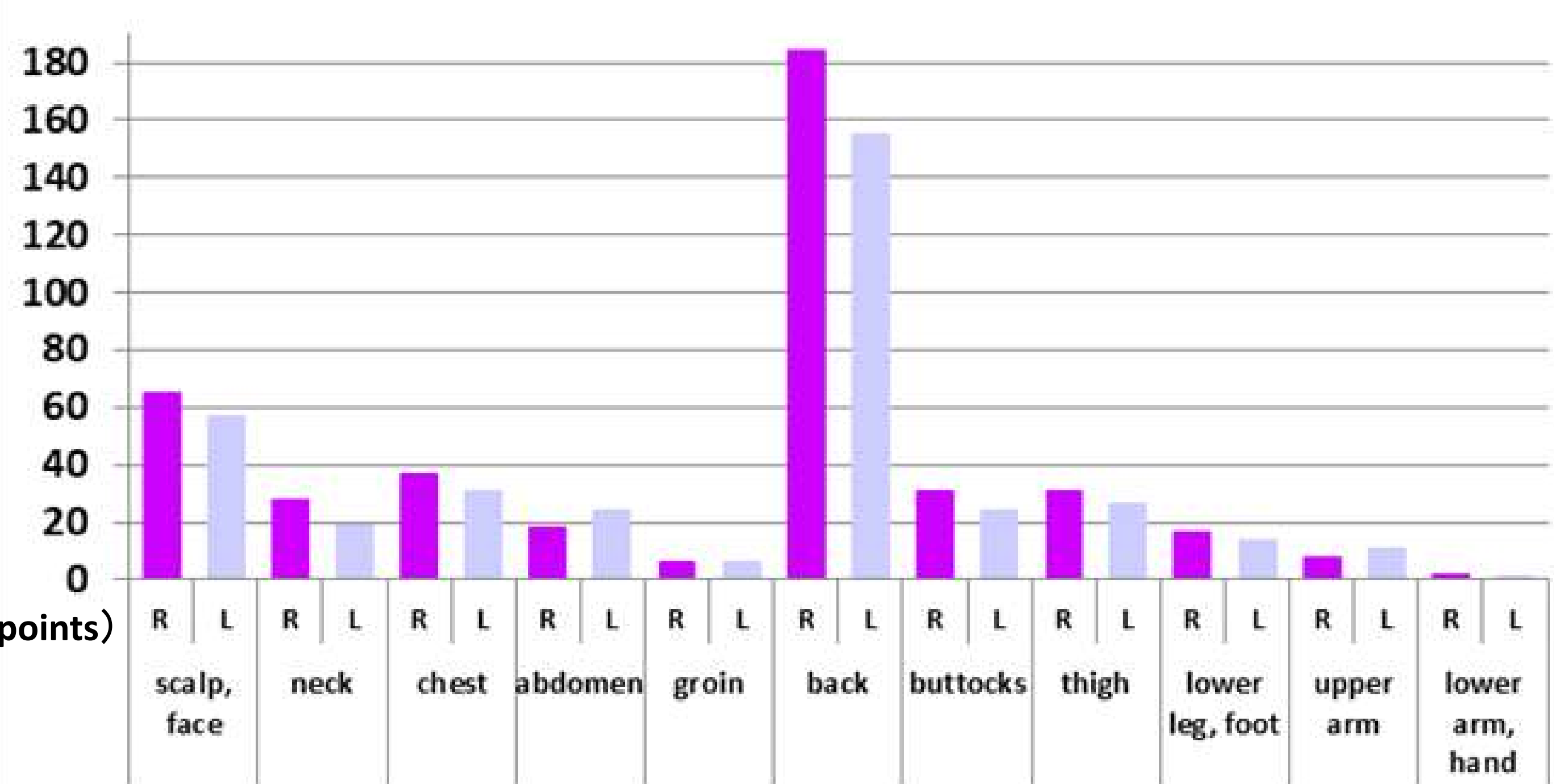

(2)Most of white macules followed Blaschko's line, although shagreen patches did not.
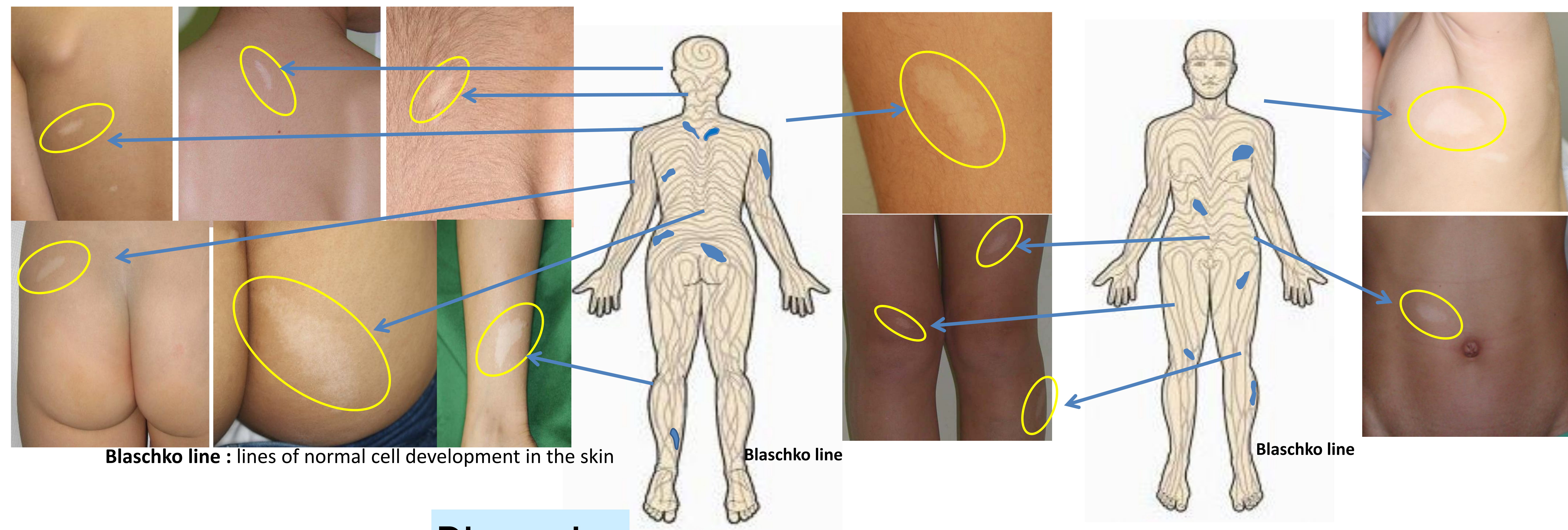

Discussion

1. Activation of mTORC1 is known to induce cell proliferation. Proliferation of fibroblasts produces collagen fibers and causes shagreen patches. We speculated, in the MTORC1 activated state, external friction activated fibroblasts and increased collagen fibers, which developed shagreen patches.

Therefore, the most common part of the shagreen patch was the friction part such as the back.

2. At an early developmental stage, precursor melanocytes start to proliferate transversally from the primitive streak along the Blaschko's lines (Fig2). Therefore we speculated that most of the white macules appeared along the Blaschko's line. We have two hypotheses for the reasons for the distributions of the white macules. The melanocytes in the white macules obtain LOH of the TSC genes. The melanocytes with LOH may exhibit both abnormal migration and abnormal melanogenesis. White macule on distal portion of the body may due to second somatic mutation of the only melanogenesis. Another hypothesis is that a second hit may occur on the melanocytes during the migration along with the Blaschko's lines. Therefore, white macules occured in a part along the Blaschko's line.

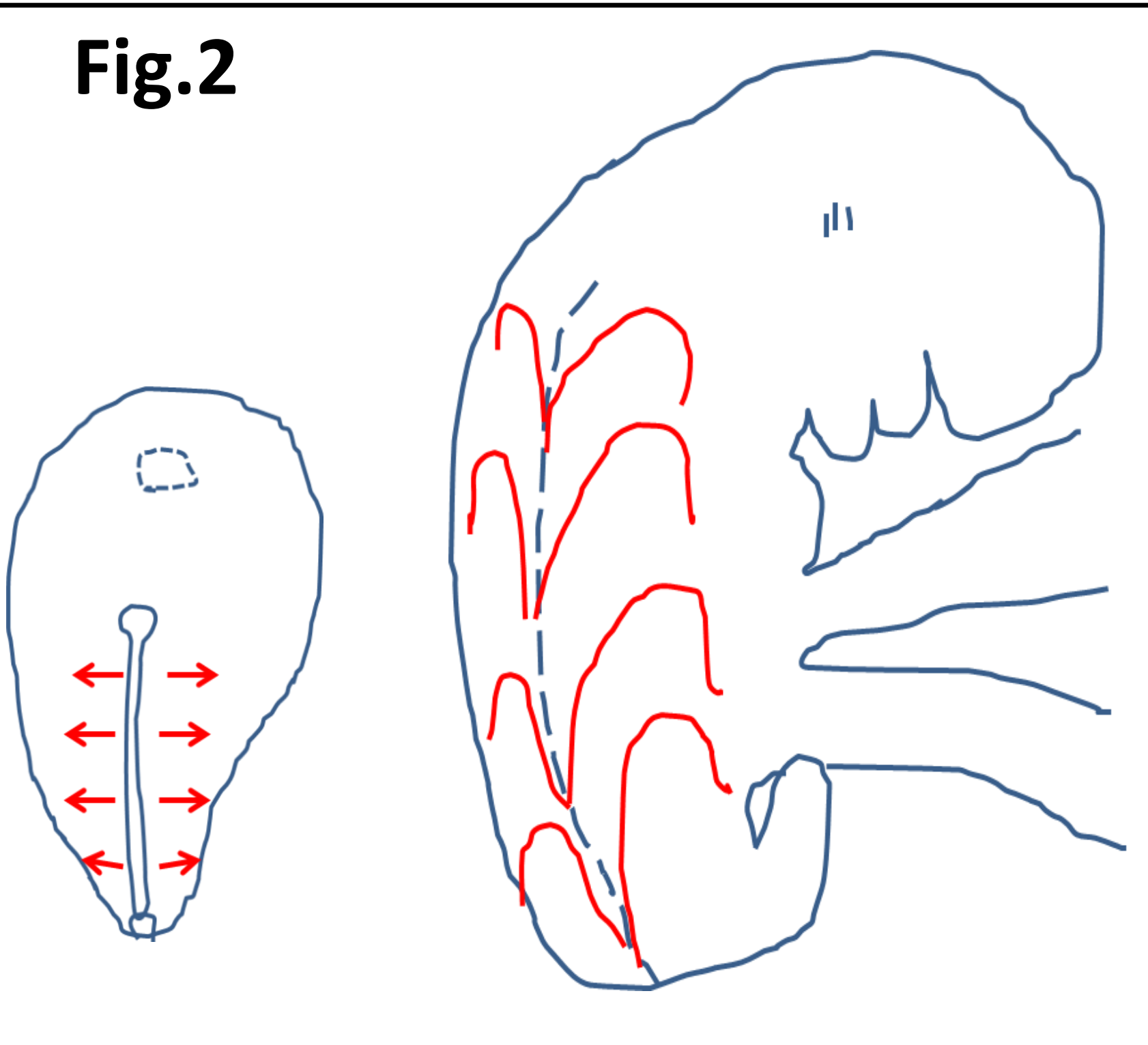

Explanation of the lines of Blaschko: At an early developemental stage precursor cells start to proliferate transversally from the primitive streak ${ }^{3)}$ 